\title{
Effects of valence on selecting and memorizing spatial information from road maps
}

\author{
Andrea Binn ${ }^{\mathrm{a},}$ * \\ ${ }^{a}$ Department of Geodesy and Geoinformation, Vienna University of Technology, Schallergasse 25/13, 1120 Vienna, Austria, \\ binnandrea@gmail.com \\ * Corresponding author
}

\begin{abstract}
Movements in unfamiliar environments require navigational tasks, which can be supported by selecting and memorizing spatial information from maps. However, this process of information extraction from maps is initiated and surrounded by a personal, emotional context. Since emotion and cognition are interlaced aspects and the visual search for information acquisition is influenced by mood and memory (Kruijne \& Meeter, 2015; Storbeck \& Clore, 2005), this research aims at determining the effects of valence on selecting and memorizing spatial information from road maps (e.g. google maps). Thereby it shall be evaluated, if common cartographic practices of visual appearance, e.g. saliency or hierarchy of objects, or the represented information itself, are in accordance with a users` need or notion of space under different emotional contexts.
\end{abstract}

Since subjective emotional contexts occur in a huge variety with low predictability and in various dimensions, this research uses a mental pre-activation of possible emotional contexts via audio-visual priming of positive and negative valence (Mayr, S., Buchner, A., 2007) before a memory task with road maps. The subsequent memory task aims at understanding "higher cognitive functions such as comprehension" (Borkin et al., 2016), by determining which information users acquire from road maps of unfamiliar space under different emotional contexts, and why they choose this information. The qualitative studies investigate on insights into the selection, learning, and recall of spatial information while being under different types of valence, such as either in an induced, acute, extrinsic stressful situation (negative valence) or in a more relaxed state (positive valence). Further they address on object selection strategies applied in the two group conditions, and on the participants' associations of object and location (Postma et al., 2004). Moreover, this research aims to contribute to a better understanding of how the emotional context affects objectlocation memory.

The results suggest that valence has an impact on the selection, learning, and recall of spatial information from road maps of unknown spaces while being under different emotional contexts. Judgements on spatial strategy, object selection and visual search strategy were affected stronger by the negative than positive arousal. Negative valence (stress) promotes strategic object selection strategies, higher memory retrieval and more accurate object-location memory, as well as a faster memory implementation of visually represented content. Positive valence promotes the development of personal associations of object and location. Findings show, that common cartographic practices (e.g. saliency, represented information) are just partly in accordance with what users select and memorize under emotional contexts.

Keywords: Priming, emotional context, selecting and memorizing spatial information from road maps

\section{References}

Borkin, M., Bylinskii, Z., Kim, N., Bainbridge, C., Yeh, C., Borkin, D., Pfister, H., Oliva, A. (2016). Beyond memorability: Visualization, recognition and recall. In: Visualization and Computer Graphics, IEEE Transactions 22, 419-528.

Kruijne, W. and Meeter, M. (2015). The long and the short of priming in visual search. Attention, Perception \& Psychophysics, 77(5), 1558-1573.

Mayr, S., Buchner, A. (2007). Negative Priming as a memory phenomenon: A review of 20 years of negative priming research. In: Journal of Psychology 1; 215, 35-51.

Postma, A, Kessels, R.P.C. van Asselen, M. (2004). The neuropsychology of object location memory. In: Human spatial memory: Remembering where. Mahwah, NJ: Lawrence Erlbaum Associates, 143-162.

Storbeck, J. and Clore, G. L. (2005). With sadness comes accuracy; with happiness, false memory: Mood and the false memory effect. In: Psychological Science 16, 785-791. 